their corresponding diameters. Duration of stay after procedure was 3-7 days and there were no adverse events post procedure such as bleeding or perforation. There were no stent migrations and stents were left in place for the rest of their life expectancy. One patient developed intermittent vomiting four weeks after stent deployment. This was possibly secondary to proximal stomach deployment, as repeated endoscopy showed a patent stent. The rest of the patients had clinical success and could tolerate diet on discharge. Range of follow up time was 1-6 months and at time of analysis GOO did not recur in all 5 patients. Three patients died due to disease progression with no symptoms of GOO prior to terminal event.

Conclusions EUS guided GJ is a novel procedure with favourable outcomes in patients with symptomatic GOO.
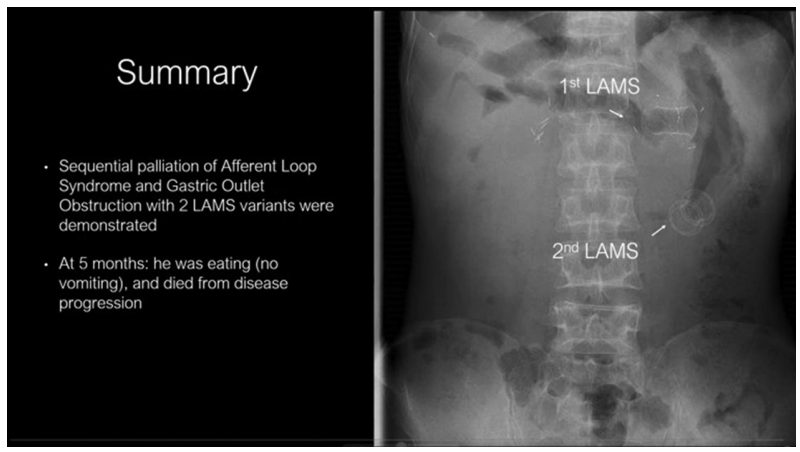

Abstract IDDF2018-ABS-0171 Figure 1

\section{IDDF2018-ABS-0172 DIETARY FACTOR RELATED TO ULCERATIVE COLITIS INFLAMMATION. AVOIDANCE OF FACTOR COULD BENEFIT DISEASE CONTROL?}

Kun Yu Tsai, Wen Sy Tsai. Division of colorectal surgery, Chang Gung Memorial Hospital, Linko, Taiwan

\subsection{6/gutjnl-2018-IDDFabstracts. 139}

Background The environment factors like western diet are considered related to development and relapse of ulcerative colitis (UC) but still lack enough supporting report. In this study, we evaluate the benefit of disease control from avoidance of possible factors.

Methods Patients with UC were enrolled between January 2006 and June 2017 and followed up for at least half year by a single colorectal specialist. Every patient was evaluated by sigmoidoscopy or colonoscopy based on Mayo endoscopic subscore (score 0-1 without rectal bleeding defined as remission). Patients were asked to avoid dairy intake at first doctor visiting. For those who had remission, we searched possible factor when relapsed and suggested avoiding the factor. The remission rate and duration were calculated to analyse.

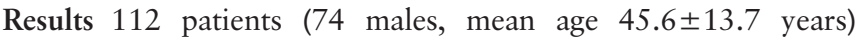

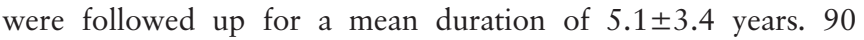
patients achieved remission at first six months. 39 patients who had no any relapse episode during the rest follow up (mean followed up $4.3 \pm 3.2$ years) were well controlled by mesalazine and avoidance of milk derivative. 49 patients had relapse and remission episode, total 97 times of relapse (mean followed up $6.5 \pm 3.2$ years). 2 patients did not achieve remission after relapse. The possible factors related to relapse were milk derivative, Chinese herb and tonic food, dietary supplement and non-dietary factor. Overall, the group with complete avoidance of possible factors has higher remission rate in 180 days compared to incomplete group ( $98 \%$ vs. $45 \%, \mathrm{p}<0.001)$. The time for achieving remission in complete avoidance group was significantly less than that in the incomplete group (45.7 \pm 35.0 days vs. $82.2 \pm 40.8$ days, $\mathrm{p}=0.022$ ). Based on this protocol, steroid use was decreased, and 15 remission patients can stop medication without relapse for $1.9 \pm 1.2$ years.

Conclusions Environment factors related to UC are multiple and might be individual. Avoid possible factor can improve UC control by decreasing steroid use and shortening the time to achieve remission. Specific Chinese herb and tonic food might be a possible factor for UC patient in Asia Area.

\section{IDDF2018-ABS-0174 FECOBIONICS: NOVEL DEFECATORY FUNCTION TEST}

Ssu Chi Chen*, Kaori Futaba, Wing Wa Leung, Cherry Wong, Tony Mak, Simon Ng, Hans Gregersen. Department of Surgery, The Chinese University of Hong Kong, Hong Kong

\subsection{6/gutjnl-2018-IDDFabstracts. 140}

Background Defecation is a complex process. Defecatory disorders may be assessed using rectal balloon expulsion test (BET), high-resolution anorectal manometry (HR-ARM) and defecography. We have developed a Fecobionics device that integrates several current tests ${ }^{1}$ to assess pressures, orientation and bending all at once by the patient bedside. The Fecobionics probe is $10 \mathrm{~cm}$ long, $12 \mathrm{~mm}$ in diameter with $8 \mathrm{~cm}$ long inflatable bag and pressure sensors at each end. It contains two gyroscopes to measure the orientation and bending during defaecation.

Aim To characterise physiological expulsion parameters in asymptomatic subjects using Fecobionics.

Methods Fecobionics was inserted into the rectum and subjects were asked to sit on a commode. The bag was filled with fluid until subjects had the sensation to defaecate. Patients were asked to expel the Fecobionics. Time to expulsion, pressures and bending angles were assessed during the expulsion. This was compared to conventional $5=\mathrm{BET}$ and HR-ARM.

Results Eight subjects (6F/2M, 50 years (25-77)) were assessed. Defaecation urge was felt at $32 \pm=$. Five subjects expelled Fecobionics in one attempt. The expulsion time for Fecobionics and BET were $32.2 \pm 6.3$ and $15.7 \pm 3.3 \mathrm{~s}$ respectively $(p<0.05)$. Linear association was found between the duration of Fecobionics and BET expulsions $\left(\mathrm{R}^{2}=0.48\right)$. During Fecobionics expulsion all pressure sensors showed elevated pressures. The front end pressure reached $20-50 \mathrm{~cm} / \mathrm{H}_{2} \mathrm{O}$ above baseline rectal pressure during anal canal passage. Rear end pressure was $28.9 \pm 4.6 \mathrm{~cm} / \mathrm{H}_{2} \mathrm{O}$ before expulsion to a maximum pressure of $144.7 \pm 15.7 \mathrm{~cm} / \mathrm{H}_{2} \mathrm{O}$ during the expulsion. Defecations could be subdivided into five distinct phases based on the front and rear pressures and their pressure difference ${ }^{1}$. The bending angle was $137.2 \pm 9.6^{\circ}$ before defaecation, $145.3 \pm 5.0^{\circ}$ at maximum contraction, and $178.7 \pm 0.9^{\circ}$ during anal passage $(\mathrm{p}<0.05)$; indicative of changes in the anorectal angle. 
Conclusions It was possible to obtain reliable data under physiological conditions using Fecobionics. Five defecatory phases could be defined by the pressure signature. Orientation and bending could also be assessed.

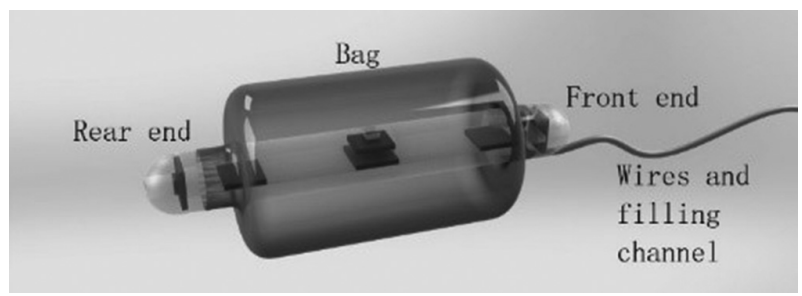

Abstract IDDF2018-ABS-0174 Figure 1

\section{IDDF2018-ABS-0175 FUNCTIONAL LUMEN IMAGING PROBE ASSESSMENT OF ANAL CANAL DISTENSIBILITY}

Ssu Chi Chen*, Kaori Futaba, Wing Wa Leung, Cherry Wong, Tony Mak, Simon Ng, Hans Gregersen. Department of Surgery, The Chinese University of Hong Kong, Hong Kong

\subsection{6/gutjnl-2018-IDDFabstracts. 141}

Background The mechanism of defaecation and continence is a complex process involving several factors. Identifying the cause of faecal incontinence is often difficult. Assessment of patients currently involve functional assessment with highresolution anorectal manometry (HR-ARM) and anatomical assessment using Endoanal ultrasound (EAUS) scan, providing limited information. EndoFLIP (Functional Lumen Imaging Probe) allows additional assessment of anal canal distensibility. 12

Aim To compare anal canal function using EndoFLIP between faecally incontinent patients (FI) and asymptomatic subjects (AS).

Methods All subjects were assessed using HR-ARM, EAUS and EndoFLIP. Using the EndoFLIP, continuous ramp distension was carried out up to $5=$ bag vol. In second ramp distension, the $5=$ volume was maintained while the subjects were asked to squeeze their anus. Anal competence of the narrowest area of the anal canal was evaluated using three distinct parameters derived from the EndoFLIP data. Mann-Whitney's U test was used for statistical analysis.

Results Sixteen FI patients (2M/14F, Mean age 61.38 years, SEM +3.34) and 9 AS (3M/6F, Mean age 57.9 years, SEM +3.69 ) were assessed. The median yield pressure (the pressure when the diameter increased from baseline) was significantly lower in the FI group at $22.99 \mathrm{cmH}_{2} \mathrm{O}\left(17.67-45.49 \mathrm{cmH}_{2} 0\right)$ compared to $55.95 \mathrm{cmH}_{2} \mathrm{O}\left(36.56-64.82 \mathrm{cmH}_{2} 0\right)$ in asymptomatic subjects. There was no significant difference in the distensibility between the FI group $0.18(0.17-0.35) \mathrm{mm} / \mathrm{cmH}_{2} 0$ compared to AS group $0.23(0.12-0.39) \mathrm{mm} / \mathrm{cmH}_{2} \mathrm{O}$ calculated as the change in diameter divided by the change in distension pressure. The squeeze strength was significantly higher in the AS group $167.3(62.0-270.8) \mathrm{mm} . \mathrm{cmH}_{2} \mathrm{O}$ compared to FI group $23.6(8.1-86.1) \mathrm{mm}_{\mathrm{cmH}} \mathrm{c}$.

Conclusions EndoFLIP demonstrated that FI patient's anal sphincters yielded at a lower pressure and had lower squeeze strength than in asymptomatic subjects, which may be clinically more relevant than squeeze pressures alone.

\section{IDDF2018-ABS-0177 THE NEW INVESTIGATION OF THE NURSING MEASURES ON ENDOSCOPIC ULTRASONOGRAPHY (EUS) FOR DEEP GASTRIC BIOPSY}

Rong Pei ${ }^{\star}$, Xiuliang Yang. Shanghai Cancer Center Hospital, China

\subsection{6/gutjnl-2018-IDDFabstracts. 142}

Background To investigate the effect of the nursing measures on endoscopic ultrasonography (EUS) for deep gastric biopsy, in order to improve the success rate and safety of biopsy.

Methods 48 patients that have underwent ordinary gastroscopy more than once. At foreign hospital and the pathological results are negative, underwent endoscopic ultrasonography deep biopsy for diagnosis in our hospital. We retrospectively analysis and summarise the importance of the preoperative perfect preparation, the high quality of nursing in operation, postoperative close observation and nursing, and every step in operation.

Results 48 cases were diagnosed by endoscopic ultrasonography. The procedure was smooth in 46 cases, 2 patients reacted with nausea and coughing. After examination, 19 patients complained of pharynx discomfort, and 1 cases had a small amount of bleeding after the operation and recovered after treatment.

Conclusions Focusing on every step and details and high-quality nursing can effectively relieve pain and improve patient compliance during ultrasonic endoscopic gastric for deep biopsy; Effective coordination of medical and nursing care can improve the positive diagnosis rate and safety of biopsy. Ensuring successful operation.

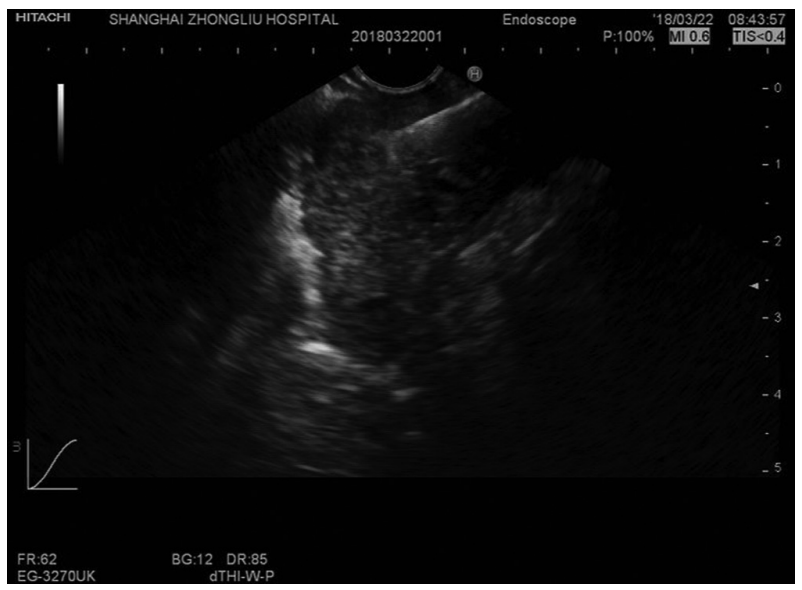

Abstract IDDF2018-ABS-0177 Figure 1

\section{IDDF2018-ABS-0179 AZATHIOPRINE: WHERE IT STANDS IN ACHIEVING NEWER MUCOSAL HEALING GOAL IN ULCERATIVE COLITIS: A COHORT STUDY}

${ }^{1}$ Rohan Badave*, ${ }^{2}$ BV Tantry, ${ }^{3}$ Sandeep Gopal, ${ }^{3}$ Suresh Shenoy. 'Senior Resident, Department of Gastroenterology, Kasturba Medical College, Mangalore, Karnataka, India; ${ }^{2}$ Professor, Department of Gastroenterology, Kasturba Medical College Mangalore, Karnataka, India; ${ }^{3}$ Associate Professor, Department of Gastroenterology, Kasturba Medical College, Mangalore, India

10.1136/gutjnl-2018-IDDFabstracts. 143 